\title{
Análise de crescimento de dois cultivares de Brachiaria brizantha (A. Rich.) Stapf. (Poaceae)
}

\author{
João Paulo Ramos Costa* \\ Liziane de Figueiredo Brito \\ Leonardo Dimas do Carmo Vieira \\ Naomi Cristina Meister \\ Carlos Henrique Oliveira Macedo \\ Pedro Luiz da Costa Aguiar Alves \\ Ana Claudia Ruggieri
}

Faculdade de Ciências Agrárias e Veterinárias

Universidade Estadual Paulista "Júlio de Mesquita Filho" CEP 14884-900, Jaboticabal - SP, Brasil

*Autor para correspondência

jpramoscosta@hotmail.com

Submetido em 18/08/2011

Aceito para publicação em 17/11/2011

\section{Resumo}

Este trabalho foi conduzido com o objetivo de estudar o crescimento e a produção primária de dois cultivares de Brachiaria brizantha através da análise de crescimento. O experimento foi realizado em casa de vegetação na Faculdade de Ciências Agrárias e Veterinárias do Campus de Jaboticabal da Unesp. Foram avaliadas as cultivares Marandu e Piatã a cada sete dias de crescimento durante dez semanas. Em cada avaliação, foram mensuradas a matéria seca total (MST) e a área foliar para estimar o índice de área foliar (IAF). Os dados de IAF e MST foram ajustados às equações e submetidos ao teste de significância para regressões. A partir das equações ajustadas foram obtidos os índices fisiológicos: taxa de crescimento da cultura (TCC), taxa de crescimento relativo (TCR), taxa de assimilação liquida (TAL) e razão da área foliar (RAF). A cultivar Piatã apresentou maior produção primária e maior eficiência no acúmulo de fotoassimilados.

Palavras-chave: Capim-marandu, Capim-piatã, Razão da área foliar, Taxa de assimilação líquida, Taxa de crescimento da cultura, Taxa de crescimento relativo.

\section{Abstract}

Growth analysis of two Brachiaria brizantha (A. Rich.) Stapf. (Poaceae) cultivars. This study was carried out with the aim of comparing the growth and primary yield of two cultivars of Brachiaria brizantha through growth analysis. The experiment was held in a greenhouse at the College of Agrarian and Veterinary Sciences in the Jaboticabal Campus of Unesp. The cultivars of Marandu and Piatã were evaluated every seven days of growth during ten weeks. At each assessment, the total dry matter (TDM) and leaf area were measured to estimate the leaf area index (LAI). The LAI and TDM data were fitted to the equations and underwent the 
test of significance for regressions. Through the adjusted equations the physiological indices were obtained: crop growth rate (CBT), relative growth rate (RGR), net assimilation rate (NAR), and leaf area ratio (LAR). The Piatã cultivar showed a higher primary yield and a more efficient accumulation of photoassimilates.

Key words: Culture's growth rate, Piatã grass, Leaf area ratio, Marandugrass, Net assimilation rate, Relative growth rate.

\section{Introdução}

Em virtude do avanço da fronteira agrícola no cerrado brasileiro, tornou-se necessária à seleção de gramíneas melhor adaptadas a essa região. Apesar da existência de muitas espécies como opção para essa exploração, as do gênero Brachiaria são as mais adaptadas ao ambiente de savana do Centro-Oeste brasileiro (ANDRADE; VALENTIM, 2007).

No ano de 1984 foi lançado no Brasil a cultivar Marandu como alternativa as pastagens de gramíneas nativas até então utilizadas. Presente em $45 \%$ das pastagens no território nacional e $50-60 \%$ das pastagens do Centro-Oeste é cultivada por apresentar adaptação e produção satisfatória (MACEDO, 2005; SANTOS FILHO, 1998).

A partir do sucesso obtido com o lançamento do Marandu, no ano de 2007 foi lançado o cultivar Piatã como mais uma alternativa para pastagens, principalmente por apresentar aptidão para pastejo diferido e produção de forragem de melhor qualidade que a Marandu (EMBRAPA, 2010).

No entanto, são escassos os estudos que comparam a produtividade primária entre cultivares de mesma espécie e as diferenças fisiológicas que determinam a adaptação e a resposta desses indivíduos a situações de cultivo e manejo (PORTES; CARVALHO, 2009; PORTES et al., 2000).

Como ferramenta de avaliação de genótipos de plantas, a análise de crescimento apresenta-se como técnica válida, pois, evidencia a influência do meio na expressão genética e agronômica, tornando possível a comparação e escolha de indivíduos específicos para cada situação (SILVA et al., 2000).

A análise de crescimento se baseia do principio de que $90 \%$ da matéria seca acumulada é advinda dos processos fotossintéticos e o restante da absorção de nutrientes minerais (BENICASA, 2003). Por meio da metodologia clássica, a estimativa de diversos índices possibilita o estudo do crescimento dos vegetais, assegurando maior conhecimento em relação à produção e os quais permitem averiguar diferenças funcionais e estruturais entre cultivares (PORTES et al., 2000; BENINCASA, 2003).

Objetivou-se com o estudo comparar o crescimento e produção de matéria seca da Brachiaria brizantha (A. Rich.) Stapf. cultivares Piatã e Marandu através da análise de crescimento.

\section{Material e Métodos}

O experimento foi conduzido em casa de vegetação na Universidade Estadual Paulista "Júlio de Mesquita Filho" Campus Jaboticabal no Departamento de Biologia Aplicada Agropecuária. As plantas foram cultivadas em vasos contendo $5 \mathrm{dm}^{3}$ de solo oriundo da camada de 0 a $20 \mathrm{~cm}$ de profundidade classificado como Latossolo Vermelho Distrófico, típico, textura argilosa, a moderado, caulinítico hipoférrico (EMBRAPA, 2006) com as seguintes características químicas: ph $(\mathrm{CaCl})=$ 5,0; M. O. $\left(\mathrm{g} / \mathrm{dm}^{3}\right)=16$; P resina $\left(\mathrm{mg} / \mathrm{dm}^{3}\right)=56 ; \mathrm{K}$ $\left(\mathrm{mmol}_{\mathrm{c}} / \mathrm{dm}^{3}\right)=2,3 ; \mathrm{Ca}\left(\mathrm{mmol}_{\mathrm{c}} / \mathrm{dm}^{3}\right)=31 ; \mathrm{Mg}\left(\mathrm{mmol}_{\mathrm{c}} /\right.$ $\left.\mathrm{dm}^{3}\right)=13 ; \mathrm{H}+\mathrm{Al}\left(\mathrm{mmol}_{\mathrm{c}} / \mathrm{dm}^{3}\right)=34 ; \mathrm{SB}\left(\mathrm{mmol}_{\mathrm{c}} / \mathrm{dm}^{3}\right)=$ 46,$3 ; \mathrm{T}\left(\mathrm{mmol}_{\mathrm{c}} / \mathrm{dm}^{3}\right)=80,3 ; \mathrm{V} \%=58$. A temperatura média e umidade no interior da casa de vegetação foram de $27,09 \pm 1,88{ }^{\circ} \mathrm{C}$ e $75,6 \pm 1,29 \%$, respectivamente, durante todo o período experimental.

Foram estudadas duas cultivares de Brachiaria brizantha: Marandu e Piatã. As sementes foram obtidas no comércio e submetidas à escarificação com ácido sulfúrico $0,1 \%$ por 2 min para a quebra de dormência e que todas germinassem ao mesmo tempo. No plantio, foi realizada adubação de 100, 360 e $200 \mathrm{mg} / \mathrm{dm}^{3}$ de $\mathrm{N}_{2}$, $\mathrm{P}_{2} \mathrm{O}_{5}$ e $\mathrm{K}_{2} \mathrm{O}$, respectivamente. A adubação de cobertura 
foi realizada 20 dias após emergência (DAE) com 60, 210 e $120 \mathrm{mg} / \mathrm{dm}^{3}$ de $\mathrm{N}_{2}, \mathrm{P}_{2} \mathrm{O}_{5}$ e $\mathrm{K}_{2} \mathrm{O}$, respectivamente. Dez DAE foi realizado o desbaste, deixando somente dez plântulas por vaso, mantendo as plântulas sadias e maiores.

As coletas foram feitas a cada sete dias, durante 10 semanas, tendo quatro vasos por semana por cultivar, consistindo cada vaso uma repetição. Em cada análise destrutiva as plantas foram separadas em pseudocolmo e lâminas foliares. As folhas tiveram suas áreas mensuradas com o auxílio do aparelho "Portable Area Meter" Licor Mod. L1 - 3000 e posteriormente foram secas em estufa a $55^{\circ} \mathrm{C}$ por $72 \mathrm{~h}$ juntamente com o pseudocolmo. O IAF foi estimado a partir da área foliar média por perfilho em cada vaso e multiplicado pela densidade populacional de perfilho (CARVALHO et al., 2006).

Os valores de MST e IAF em função do tempo(DAE) foram submetidos à ANOVA, ajustados as equações matemáticas e submetidos ao teste de significância para as regressões com o programa computacional AJUSTE (ZULLO JUNIOR; ARRUDA, 1987). Os modelos foram escolhidos com base na significância dos coeficientes de regressão e no coeficiente de determinação. Os índices fisiológicos de taxa de crescimento da cultura (TCC), taxa de crescimento relativo (TCR), taxa de assimilação liquida (TAL) e razão da área foliar (RAF) foram estimados a partir das equações previamente ajustadas utilizando o programa ANACRES (PORTES; CASTRO JUNIOR, 1991).

\section{Resultados e Discussão}

Os cultivares apresentaram crescimento similar entre si. A MST e o IAF foram crescentes em função dos DAE e melhor se ajustaram às equações do tipo exponencial quadrática conforme mostrado na Tabela 1.

A taxa de crescimento da cultura (TCC) apresentado na Figura 1 foi crescente até se tornar máxima aos 42 DAE para as cultivares em estudo. No entanto, a cultivar Marandu apresentou menor taxa de crescimento $(0,168 \mathrm{~g} /$ $\mathrm{m}^{2} /$ dia) quando comparada ao Piatã, que apresentou máxima TCC de $0,170 \mathrm{~g} / \mathrm{m}^{2} /$ dia nessa mesma idade. No período em que a TCC é máxima, segundo Kvet et al. (1971), o IAF é ótimo, e a fotossíntese é máxima uma vez que o sombreamento é mínimo, assim, otimizando a fotoassimilação. Apesar do IAF ótimo dacv. Marandu ser maior $(1,36)$ em relação ao Piatã $(1,28)$, a produção primária (TCC) foi menor.

TABELA 1: Coeficientes (C, B e A) das equações $\mathrm{Y}=$ $\mathrm{C}^{*} \mathrm{EXP}\left[(\mathrm{B})^{*} \mathrm{DAE}+(\mathrm{A}) \mathrm{DAE}^{2}\right]$ ajustadas aos dados de matéria seca total (MST) e índice de área foliar (IAF) em função do tempo (Dias após emergência) das cultivares Marandu e Piatã.

\begin{tabular}{cccccccc}
\hline \multirow{2}{*}{ Cultivar Variável } & \multicolumn{4}{c}{ Coeficientes da equação } & & P \\
\cline { 3 - 6 } & & C & B & A & & Ralue \\
\hline \multirow{2}{*}{ Marandu } & MST & $-0,0013862690$ & 0,16535460 & 0,03836328 & 0,99 & $<0,01$ \\
& IAF & $-0,0003822930$ & 0,07873056 & 0,10394660 & 0,99 & $<0,01$ \\
& MST & $-0,0012653980$ & 0,15536890 & 0,04744770 & 0,99 & $<0,01$ \\
\multirow{2}{*}{ Piatã } & IAF & $-0,0003842517$ & 0,07542916 & 0,10406370 & 0,99 & $<0,01$ \\
\hline
\end{tabular}

FIGURA 1: Taxa de crescimento da cultura (TCC) das cultivares Marandu e Piatã em função dos dias após a emergência (DAE).

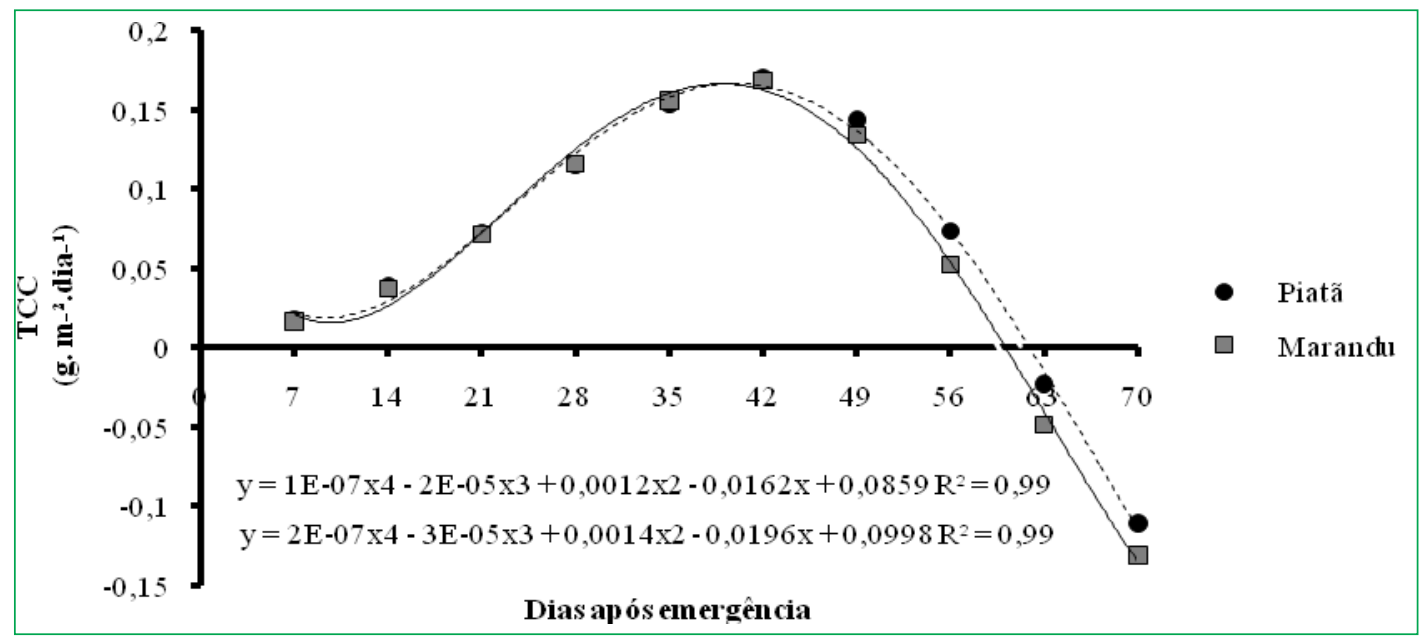


A partir dos 42 DAE a análise da TCC para as duas cultivares demonstrou que o ganho em matéria seca foi diminuído e o acúmulo estabilizado aos 59 e 61 DAE para o Marandu e Piatã respectivamente. A partir dessas idades a TCC apresentou-se negativa. Segundo Portes e Carvalho (2009), esse comportamento é devido ao aumento do sombreamento nas folhas basais, dessa forma, aumentando a taxa de senescência, tornando-a maior que a taxa de crescimento de novos tecidos.

Comportamentos semelhantes foram encontrados por Euclides et al. (2008), avaliando o acúmulo de matéria seca das cultivares de Brachiaria brizantha a campo. As duas cultivares apresentaram produção semelhante, no entanto a Piatã com $3.850 \mathrm{~kg} /$ ha foi ligeiramente maior e a Marandu sendo a menos produtiva, já que apresentou produção de $3.730 \mathrm{~kg} / \mathrm{ha}$.
$\mathrm{Na}$ Figura 2, verifica-se que a cv. Marandu apresentou maior TCR no período de 7 a 28 DAE, enquanto que a partir dos 42 DAE a cultivar Piatã apresentou maior TCR quando comparado com o cv. Marandu, embora os dois cultivares apresentarem TCR decrescente, o que é natural ao longo da ontogenia da planta (REYES-CUESTA et al., 1995). Em parte, esse processo é resultante do aumento da proporção de tecidos não fotossintetizantes.

No entanto, a partir dos 42 dias a cv. Piatã mostrouse mais eficiente na produção de matéria seca já que, segundo Silva et al. (2000), a taxa de crescimento relativo de uma planta é a capacidade de produção de matéria seca a partir da já existente.

A TAL das cultivares foram crescentes dos 7 DAE até atingir o máximo aos 28 (Figura 3), desse período

FIGURA 2: Taxa de crescimento relativo (TCR) das cultivares Marandu e Piatã em função dos dias após a emergência (DAE).

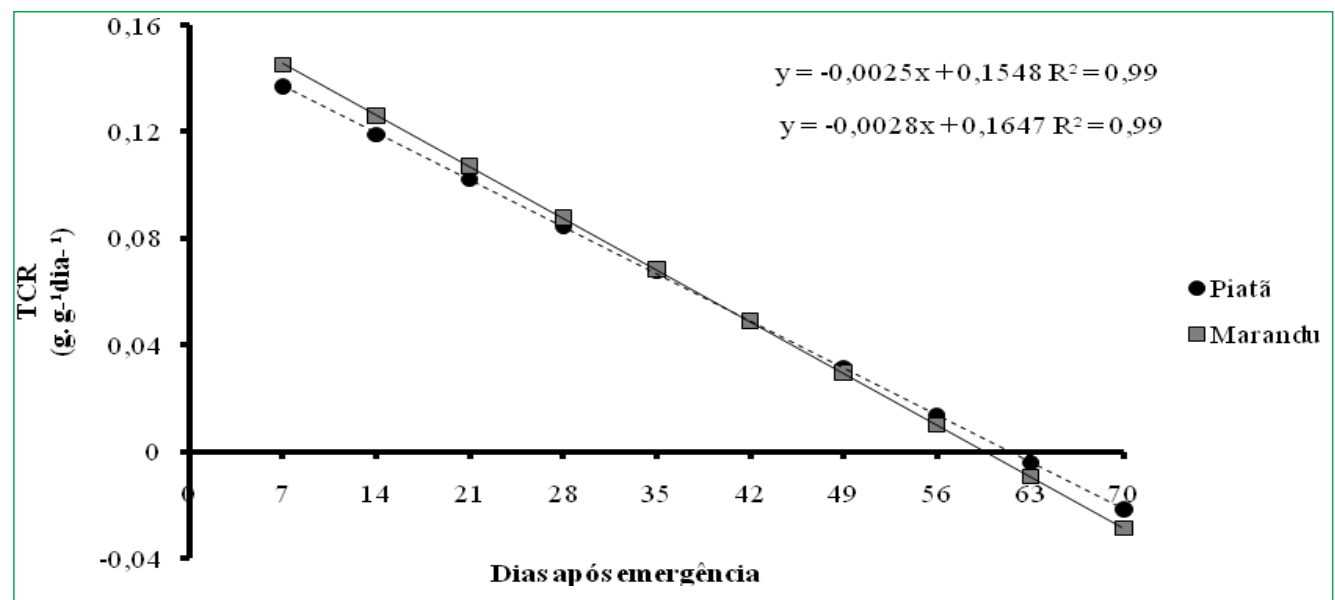

FIGURA 3: Taxa de assimilação liquida (TAL) das cultivares Marandu e Piatã em função dos dias após a emergência (DAE).

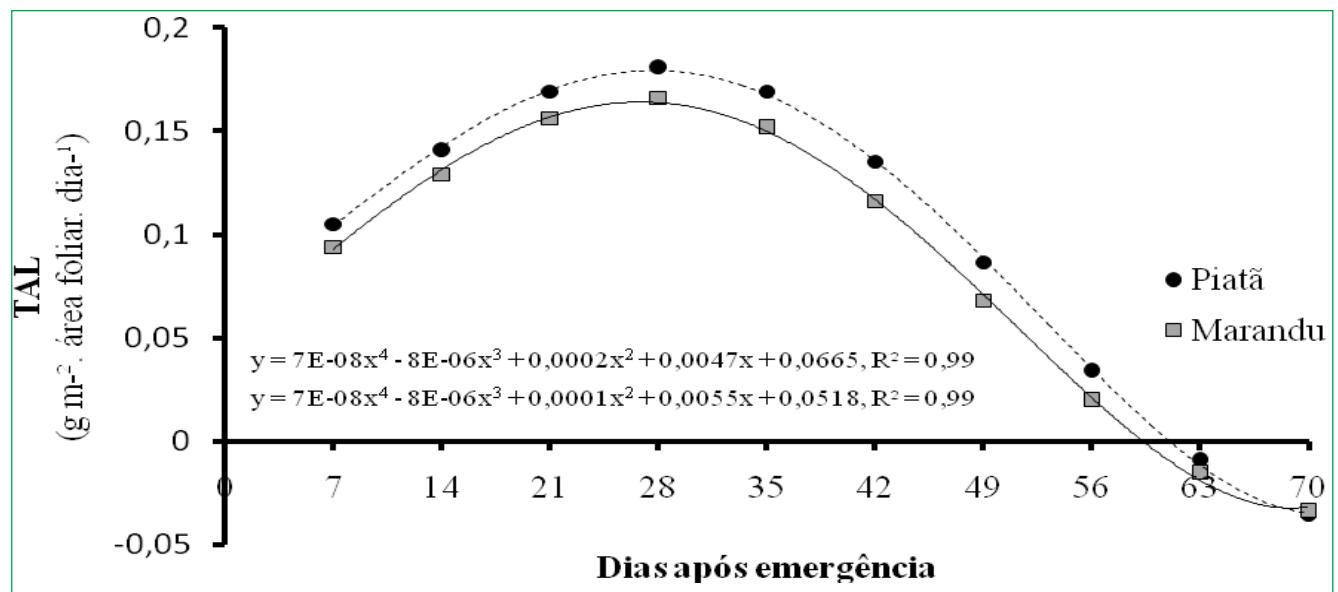


até a última avaliação apresentou-se decrescente para os dois cultivares. O decréscimo apresentado pelas cultivares pode ser explicado pela redução da eficiência fotossintética que acontece com o avanço da idade das folhas (PARSONS et al., 1983). Sobretudo, em todo período de crescimento o ganho em matéria seca por unidade de área foliar da cultivar Piatã foi superior ao Marandu. Portanto, o Piatã mostra ser mais eficiente no acúmulo de matéria orgânica quando comparado ao Marandu.

Conceitualmente, a TAL é a medida da eficiência fotossintética de uma planta e representa o balanço do material produzido pela fotossíntese e o que foi perdido pela respiração (SILVA et al., 2000). Indivíduos que apresentam baixa perda de carbono através da respiração são preferenciais em programa de seleção de novas cultivares forrageiras, pois, apresentam menor custo com a respiração e frequentemente são mais produtivos (PILBEAM, 1992).

As TALs foram negativas aos 61 e 59 DAE para a Marandu e Piatã, respectivamente. Segundo Gomide et al. (2003), essa resposta da planta é resultante do aumento da demanda respiratória pelas folhas velhas, que antes contribuíam na produção de fotoassimilados e com o avanço do sombreamento e idade, a folha assume o papel de dreno, diminuindo a eficiência fotossintética da planta.

A RAF (Figura 4) do Marandu foi maior durante todo período de crescimento. Embora a capacidade fotossintética do Marandu ter se apresentado superior em todo o período de crescimento, a produtividade primária não foi maior que a do Piatã, o que sugere a baixa eficiência do Marandu no acúmulo de massa seca uma vez que possui maiores órgãos fotossintéticos quando comparado com o Piatã.

A RAF dos cultivares diminuíram da primeira avaliação até os 42 DAE. Andrade et al. (2002) explicam que o decréscimo é atribuído ao investimento de fotoassimilados para desenvolvimento de órgãos não fotossintetizantes como colmos, bainha e raiz, tornando-a mais ineficiente já que a massa total aumenta e a capacidade fotossintética pouco varia.

As cultivares em estudo são extensamente utlizadas na pecuária brasileira. Diante da grande importância que apresenta para o segmento de produção de ruminantes a pasto, estudos foram realizados com intuito de averiguar a aptidão dessas cultivares. Euclides et al.,(2009) estudando a produção de bovinos de corte em pastagem de capim-Piatã e Marandu, reporta que a cultivar Piatã é preferencial para o ganho de peso quando comparado com o Marandu, isso porque apresentou maior ganho de peso médio diário e maior produção de matéria seca por hectare. Em outro trabalho realizado por Euclides et al., (2008) a cv. Piatã apresenta maior taxa de acúmulo de lâmina foliar em relação à cv Marandu, o que condiz com os resultados encontrados no presente trabalho.

Conclui-se que os cultivares possuem crescimento semelhante, no entanto, o Piatã apresentou maior

FIGURA 4: Razão da área foliar (RAF) das cultivares Marandu e Piatã em função dos dias após a emergência (DAE).

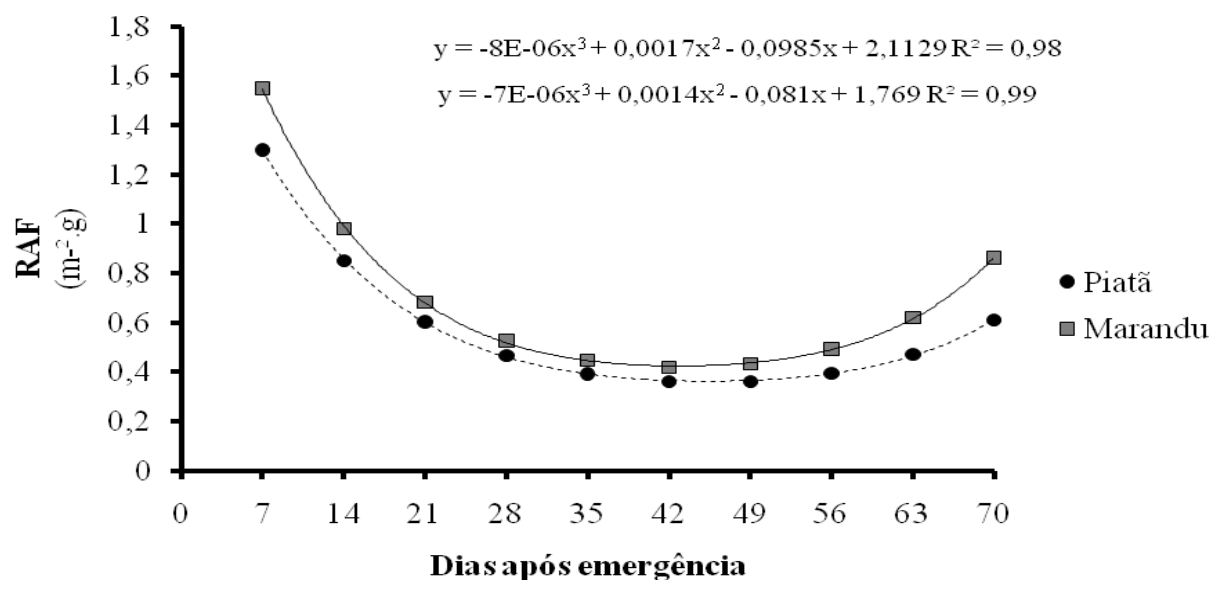


produção primária e maior eficiência no acúmulo de fotoassimilados, mostrando ser mais produtiva que a cultivar Marandu.

\section{Referências}

ANDRADE, A. C.; FONSECA, D. M.; LOPES, R. S.; NASCIMENTO-JUNIOR, D.; GOMIDE, J. A.; CECON, P. R.; QUEIROZ, D. S.; PEREIRA, D. H.; CARDOSO, R. C. Análise de crescimento do capim-elefante napier adubado e irrigado. In: REUNIÃO ANUAL DA SOCIEDADE BRASILEIRA DE ZOOTECNIA, 39, 2002, Recife. Anais... Recife: SBZ, 2002. CDROM.

ANDRADE, R. P.; VALENTIM, J. F. Síndrome da morte do capim-brizantão no Acre: características, causas e soluções tecnológicas. (Documentos, 105). Rio Branco: EMBRAPA ACRE, 2007. 43 p.

BENINCASA, M. M. P. Análise de crescimento de plantas: noções básicas. 2 ed. Jaboticabal: Funep, 2003. 41 p.

EMBRAPA. Sistema brasileiro de classificação de solos. Seminário Nacional de Levantamento e Conservação de Solos. 2 ed. Brasília: EMBRAPA, 2006. 306 p.

EUCLIDES, V. P. B.; MACEDO, M. C. M.; VALLE, C. B.; DIFANTE, G. S.; BARBOSA, R. A.; CACERE, E. R. Valor nutritivo da forragem e produção animal em pastagens de Brachiaria brizantha. Pesquisa Agropecuária Brasileira, Brasília, v. 44, n.1, p. 98-106, 2009.

EUCLIDES, V. P. B.; MACEDO, M. L. C. M.; VALLE, C. B.; BARBOSA, R. A.; GONÇALVES, W. V. Produção de forragem e características da estrutura do dossel de cultivares de Brachiaria brizantha sob pastejo. Pesquisa Agropecuária Brasileira, Brasília, v. 43, n. 12, p. 1805-1812, 2008.

GOMIDE, C. A. M.; GOMIDE, J. A.; ALEXANDRINO, E. Índices morfogênicos e de crescimento durante o estabelecimento e a rebrotação do capim-mombaça (Panicum maximum Jacq.). Revista Brasileira de Zootecnia, Viçosa, v. 32, p. 795-803, 2003.

KVET, J.; ONDOCK, J. P.; NECAS, J.; JARVIS, P. G. Methods of growth analysis. In: SESTAK, Z.; CATSKY, J.; JARVIS, P. G. (Eds.). Plant photosynthetic production: manual of methods. Haia: N. V. Publishers, 1971. p. 343-349.

CARVAlHO, C. A. B.; PACIULLO, D. S. C.; ROSSIELlO, R. O. P.; DERESZ, F. Dinâmica do perfilhamento em capim-elefante sob influência da altura do resíduo pós-pastejo. Pesquisa Agropecuária Brasileira, Brasília, v. 41, p. 145-152, 2006.
EMBRAPA. Capim Piatã. 2007. Disponível em <HTTP://www. cnpgc.embrapa.br/produtoseserviços/pdf/piata.pdf $>$. Acesso em: 20 maio 2010 .

MACEDO, M. C. M. Pastagens no ecossistema cerrado: evolução das pesquisas para o desenvolvimento sustentável. In: REUNIÃO ANUAL DA SOCIEDADE BRASILEIRA DE ZOOTECNIA, 42, 2005, Goiânia. Anais... Goiânia: SBZ, 2005. p. 56-84.

PARSONS, A. J.; LEAFE, E. L.; COLLETT, B.; STILES, W. The physiology of grass production under grazing: I - characteristics of leaf and canopy photosynthesis of continuously grazed sward. Journal of Applied Ecology, London, v. 20, p. 117-126, 1983.

PILBEAM, C. J. Effect of nitrogen supply on the growth and senescence of leaves of Lolium perenne with contrasting rates of leaf respiration. Annals of Botany, Oxford, v. 70, p. 365-370, 1992. PORTES, T. A.; CASTRO JÚNIOR, L. G. Análise de crescimento de plantas: um programa computacional auxiliar. Revista Brasileira de Fisiologia Vegetal, Campinas, v. 3, p. 53-56. 1991.

PORTES, T. A.; CARVALHO, S. I. C.; OLIVEIRA, I. P.; KLUTHCOUSKI, J. Análise do crescimento de uma cultivar de braquiária em cultivo solteiro e consorciado com cereais. Pesquisa Agropecuária Brasileira, Brasília, v. 35, p. 1349-1358, 2000.

PORTES, T. A.; CARVALHO, S. I. C. Crescimento e alocação de fitomassa de cinco gramíneas forrageiras em condições de cerrado. Revista de Biologia Neotropical, Goiânia, v. 6, p. 1-14, 2009.

REYES-CUESTA, R.; LOPES, N. F.; OLIVA, M. A.; FRANCO, A. A. Crescimento e conversão da energia solar em Phaseolus vulgares em função da fonte de nitrogênio. Revista Ceres, Viçosa, v. 42, p. 405-455, 1995.

SANTOS FILHO, L. F. Producción de semillas: el punto de vista del sector privado brasileño. In: MILES, J. W.; MAASS, B. L.; VALLE, C. B. (Ed.). Brachiaria: biología, agronomía y mejoramiento. Cali: CIAT, 1998. p. 156-162.

SILVA, L. C.; BELTRÃO, N. E. de M.; AMORIM NETO, M. da $\mathrm{S}$. Análise de crescimento de comunidades vegetais. Campina Grande: EMBRAPA-CNAPA, 2000. 47 p. (Circular Técnica, 34).

SOARES FILHO, C. V. Recomendação de espécie e variedade de Brachiaria para diferentes condições. In: SIMPÓSIO SOBRE MANEJO DE PASTAGEM, 11, 1994, Piracicaba, Anais... Piracicaba: FEALQ. 1994. p. 25-48.

ZULLO, J. R. J.; ARRUDA, F. B. Programa computacional para ajuste de equações em dados experimentais. Campinas: Instituto Agronômico de Campinas, 1987. 31 p. (Boletim Técnico, 113). 